\title{
SIMULATION OF THE «COSMONAUT-ROBOT»SYSTEM INTERACTION ON THE LUNAR SURFACE BASED ON METHODS OF MACHINE VISION AND COMPUTER GRAPHICS
}

\author{
B. I. Kryuchkov ${ }^{\text {a }}$, V. M. Usov ${ }^{\text {a }}$, V. A. Chertopolokhov ${ }^{\text {b, } * \text {, A. L. Ronzhin }}{ }^{\text {, }}$, A. A. Karpov ${ }^{\text {c }}$ \\ ${ }^{a}$ Yu. Gagarin Research \& Training Cosmonauts Center, Star City Moscow Region, Russia - (b.kryuchkov, v.usov)@gctc.ru \\ ${ }^{\mathrm{b}}$ Lomonosov Moscow State University, Mathematical-Mechanical Faculty, Moscow, Russia - psvr.msu@gmail.com \\ ${ }^{c}$ St. Petersburg Institute for Informatics and Automation of the Russian Academy of Sciences, St. Petersburg, Russia - \\ (ronzhin, karpov)@iias.spb.su
}

\author{
Commission II, WG II/5
}

KEY WORDS: Lunar Exploration, Extravehicular Activity (EVA), Human-Robot Interaction (HRI), Mobile Robot Control, "Follow Me" Mode, Gesture Interface, Object Tracking, Gesture Recognition, Motion Capture

\begin{abstract}
:
Extravehicular activity (EVA) on the lunar surface, necessary for the future exploration of the Moon, involves extensive use of robots. One of the factors of safe EVA is a proper interaction between cosmonauts and robots in extreme environments. This requires a simple and natural man-machine interface, e.g. multimodal contactless interface based on recognition of gestures and cosmonaut's poses. When travelling in the "Follow Me" mode (master/slave), a robot uses onboard tools for tracking cosmonaut's position and movements, and on the basis of these data builds its itinerary. The interaction in the system "cosmonaut-robot" on the lunar surface is significantly different from that on the Earth surface. For example, a man, dressed in a space suit, has limited fine motor skills. In addition, EVA is quite tiring for the cosmonauts, and a tired human being less accurately performs movements and often makes mistakes. All this leads to new requirements for the convenient use of the man-machine interface designed for EVA. To improve the reliability and stability of human-robot communication it is necessary to provide options for duplicating commands at the task stages and gesture recognition. New tools and techniques for space missions must be examined at the first stage of works in laboratory conditions, and then in field tests (proof tests at the site of application). The article analyzes the methods of detection and tracking of movements and gesture recognition of the cosmonaut during EVA, which can be used for the design of human-machine interface. A scenario for testing these methods by constructing a virtual environment simulating EVA on the lunar surface is proposed. Simulation involves environment visualization and modeling of the use of the "vision" of the robot to track a moving cosmonaut dressed in a spacesuit.
\end{abstract}

\section{INTRODUCTION}

A design of Human Robot Interaction (HRI) in relation to robots-assistants attracted the attention of researchers not only in the obvious aspects of practical utility, but also in the light of solving actual scientific problems of developing a humanmachine dialogue that meets the requirements of familiarity, availability and simplicity.

A new stage of development of contactless methods of interactions is connected with the inclusion of robots-assistants in the professional activity of the person (operator) in extreme environments (Karpov et al., 2016; Kryuchkov et al., 2014, Kryuchkov et al., 2015; Burridge, Graham, 2001). The purpose and the advantages of robots-assistants in this case are fairly obvious: a person receives more favorable conditions for operating in extreme conditions, it facilitates the exploration of a poorly-studied environment, operator may spend less time in hazardous conditions, but the problem of safety in a joint operation of humans and robots still remains. In a certain sense, the problem goes to a higher level of complexity (the need of a high-quality information provision) and requires new technological approaches.
In this paper, the questions of information providing for cosmonauts are considered in respect to the gesture-type control of mobile robots during extravehicular activity (EVA) on the lunar surface (Burridge, Graham, 2001; Liu, Luo and Ju, 2016), with taken into account a set of human factor constraints in EVA. The task of modeling such a system of information support in the laboratory conditions is consist of designing interfaces with the greatest possible similarity for the real conditions, based on the description of multi-level system for monitoring the activities of humans and robots in EVA.

\section{FEATURES OF EVA WITH REGARD TO THE CONDITIONS ON THE LUNAR SURFACE}

Organization of interaction between an operator and a mobile robot is often performed on a contactless basis. Among other things, the choice of method of communication is determined by the characteristics of the perception of the operator and his ability to encode heterogeneous information (Vorona et al, 2016). An important question is which means of information support in complex and extreme conditions are preferable based on the human factors restrictions. The perception of information by the operator depends substantially on the form in which data is presented and displayed and how easy it is to identify and

\footnotetext{
* Corresponding author
} 
transcode signals and messages. In cases when the information comes through different channels of communication and is designed for different modalities of perception, it is important to ensure its consistency and coherence with real events at the time of receipt by the operator (Vorona et al, 2016). Another aspect of the security task relates to the requirement of relevance of data and timing of information receipt synchronization for all participants of communication (Karpov et al., 2016; Kryuchkov et al., 2014).

When developing gesture-type control for robots on the lunar surface need to consider the experience of EVA in orbital flights to the ISS in relation to the role and task of all crew members to ensure security and mutual insurance. EVA is one of the most complex and energy-consuming activities of the cosmonaut. For the EVA on the ISS were developed stringent safety regulations, which should be taken into account during EVA on the lunar surface.

The functions of a robot may consist of carrying cargo and tools, exploring the terrain, creating and updating of digital maps, collecting and transmitting of navigation data and data on the status of onboard systems through telemetry channels, which then sent to the Command Centre of the lunar EVA unit. In addition, a robot may collect medical data from a space suit control system and from a system of operative medical monitoring of the health of the cosmonaut during EVA. The functions of a robot-assistant can also include monitoring of operator activity, informing the observer, controlling the efficiency and safety of EVA in general, transporting (medical evacuation) a damaged human to the lunar base, including the mode of maintenance of vital functions (primarily cardiorespiratory).

In this paper special attention is given to the interaction between an operator and a robot-assistant on the lunar surface. With regard to the joint exploration of the territory of the lunar surface it is proposed to perform control mode "Follow me" with gesture control. This mode is not the only possible when using a robot-assistant, however it is especially complicated in implementation and critically important in aspects of interactive communication security. Among other uses of the robotassistant, we can specify: construction of the route of the reverse movement to the place of dislocation along the explored trajectory; moving in a circle for a panoramic view of the shooting area (or video-photography presentations).

Thus, to ensure safety and facilitate human interaction with a mobile robot in terms of EVA for remote gesture control we propose the use of two coherent and synchronized real-time component of information support:

1) For the cosmonaut - Manager - to display the current situation based on a visual 3D representation of the virtual desktop in a multi-mode technology for the "outside observer" or based on a multifunctional display, working in different modes (including direct observation of the picture on the monitors of computer vision of a robot, the synthesized pattern of the recognized gestures, electronic chart, symbolic representation of teams on the move and the current motion parameters, etc.).

2) For the operator in "a team on lunar surface" - the information management interface supports gesture control for the movement of the robot; in this case the focus of the study should be on the circuit of visual feedback. This requires to use a signal indicator, placed directly on the suit, which gives the operator the ability to: a) correct recognition of robot gesture commands; b) ensure that the specified distance of the joint movement is respected; c) perform spatial orientation for targeting reference points on the electronic map; d) receive timely notification of danger in terms of the prevention of dangerous rapprochement with the surrounding objects, including other mobile robots.

The construction of the interaction, that were described above for the EVA, as the complex technical-organizational system, involves the use of multi-component interface is substantially focused on the information needs of all active participants in the EVA. Consider the possibility of decision-making and information needs of participants in the above-described interaction "crew-robots" during the EVA. Pre-analyze what the on-Board equipment for a mobile robot is required to navigate in order to provide participants with EVA all the necessary information.

\section{INFORMATION SUPPORT OF MOBILE ROBOTS IN AUTONOMOUS MODE AND AT JOINT PERFORMANCE EVA WITH OTHER PARTICIPANTS}

The on-board equipment of mobile robots for navigating on the Moon and for providing cosmonauts with the information about the current situation deserves special consideration since the high level of sensory capabilities and artificial intelligence of the mobile robot (model of the external world) guarantees the possibility of moving in Autonomous mode, in joint motion control mode with the operator and ensures safety.

It is assumed that the avionics of a robot-assistant will include communication modules, positioning and navigation modules, digital maps, sensors of control, detection and tracking of moving objects, and, in the context of contactless interaction, recognition of gestures and postures of the upper part of the human's body. Requirements for on-board equipment and sensors of the robot for the "Follow me" mode can be formulated on the basis of the following list of functions: liaising with the operator, based on gesture recognition through the tracking systems of moving objects, maintaining radio communication to receive commands group managers (the head of operations) and transfer mode telemetry at lunar module about self-diagnostics of the health of onboard systems, identification of control commands, a video monitoring environment, as well as data on the positioning and medical telemetry according to the system of medical supervision and monitoring system of environmental parameters inside the spacesuit.

Proceeding from the presented list of functions, a mobile robot must have on-board equipment that meets certain requirements. First of all, this telemetry system, which is designed to transmit to the Central unit the information about working capacity of the robot, the navigation parameters, the results of detection and tracking moving objects, etc.

To support the navigation system of the robot required to provide the cosmonauts visual feedback (photos and/or video) obtained by using computer vision of the robot, to use operational data to build feedback on positioning based on ranging, the detection of moving objects and their localization ("sensors-e-cards"), and feedback for situational reaction as a result of monitoring the status of the health of on-board systems, accounting status of work in team or independently, of the pending status of the team, its performance or failure. In addition, the actual question of the ability to control machine vision robot sensors and sensors to control the distance from the 
reference points and method of signal processing for visualization, including the choice of the coordinate system for the representation of the relative position of the controlled objects is also relevant. The artificial intelligence of the robot and its control system must be able to maintain the safety of the robot in Autonomous mode. For this you need to respond to obstacles, the ability to avoid collisions, to prevent the rollover. The most difficult situation can arise in conditions of communication failure, delays and signal transmission when communication is unreliable. All such situations must be clearly recognized, and the robot has to promptly inform the operator. We cannot exclude the loss of function of the operator. This situation requires the implementation of means of control of the functional state of health and medical telemetry, and analysis of behavioral activity, the nature of the issued command, the recognition of the reasons for the termination of interaction between a human and a robot, etc.

\section{METHODS OF TRACKING THE MOVEMENTS OF A COSMONAUT}

Visual tracking methods are becoming more and more popular due to their simplicity and low cost compared to other methods. Although the most commonly used procedure to capture quantitative movement data is the use of attached markers or patterns, but markerless tracking is seen as a potential method to make the movement analysis quicker, simpler and easier to conduct.

There are many advantages of markerless tracking compared to marker-based tracking. First, there are no markers that can be placed incorrectly or can lead to inaccuracy because of metal reflection artefacts. Second, much time can be saved if no markers have to be attached. However, in order for the markerless approach to become a standard in movement analysis it has to be accurate and reliable.

Nowadays, markerless motion capture (MoCap) methods for the estimation of human body kinematics are at the leading edge of tracking technologies. Over the past few years, those technologies have advanced drastically. There are two primary markerless tracking approaches: feature-based, required single capture camera, and z-buffer-based, required several capture cameras. To implement feature-based tracking, one have to apply image processing methods to improve the quality of the image and mathematical algorithms to find joints, but it presumes that the tracked object is seen well. Another approach is highly robust to topology changes and overlapping, it also supports incremental reconstruction, improving the surface estimation over time, as well as parameterizing the non-rigid motion.

The optical system of the motion capture suit to perform monitoring tasks in real time, but have a significant drawback they are susceptible to errors in human movement. To resolve this issue, hybrid tracking techniques are used when the readings of optical sensors supplement the indications of autonomous inertial sensors.

\section{POSSIBILITIES AND LIMITATIONS OF THE GESTURAL INTERFACE WHEN A COSMONAUT WORKS IN A SPACESUIT}

Data obtained with the system of tracking used in the unit of the gesture recognition. The gesture interface in the context of the EVA on the lunar surface need to be considered taking into account features of perception of a person working in extreme environment and the impact of the fact that the use of individual means of rescue, protection of the body and creation of favorable conditions for the human's organism.

For example, the history of the development of a space suit can be traced, expanding its operational capabilities and ease of use. However, the necessary compromise between the protective properties of the suit and its operational capabilities, primarily, the question of the limits of the cosmonaut in the amount of limb movements (e.g. decrease in the number of degrees of freedom in the links of the shoulder-arm-wrist-fingers), strenuous exercise in the performance of many types of movements, inability to use fine motor finger gloves suits etc.

These limitations lead to the need for recognition of gestures to focus on the upper body. In addition, the glazing of the front part of the helmet of the suit determines the possibility of the review and affects the maintenance of spatial orientation and visual possibilities of determining the characteristics of the terrain. That is, visual gesture controls performed in full only in a certain position of the hands. In addition, from the practice are known cases of poor coordination and visual-motor disorders after exposure to altered gravity, in particular, in zero gravity, which can be a risk factor for cosmonauts of the lunar missions.

\section{GROUND-BASED SIMULATION OF EVA, A COMPARISON OF THE CAPABILITIES OF FIELD AND LABORATORY SIMULATION}

Performance evaluation of the considered scheme of interaction of humans and robots during the EVA and the adequacy of their information security requires the development of modeling software and hardware system that implements methods for capturing and tracking moving objects, motion capture of human hands, posing and navigation of mobile robots and synthesis of the indicators of the parameters of the current situation in the various forms of display information, including the use of the panorama display, information frames multifunctional indicators and specific command devices of the type wearable signal indicators.

With regard to extreme robotics this list of engineeringpsychological questions requires in-depth research, which is necessary to create a computer simulation of the complexes with the corresponding instrumental and methodological equipment (Karpov et al., 2016; Damian et al., 2013; Liu, Luo, and Ju, 2015).

In the faculty of mechanics and mathematics of the Lomonosov Moscow State University created a stand and tools to apply virtual reality technology for testing and modeling of operator interaction with a mobile robot on the surface (see Figure 1). For immersion of the operator in a simulated environment the visualization on the basis of the virtual reality helmet is used. The input to the visualization system gives information about the current state of the mathematical model of the robot and its surrounding objects, which allows us to simulate a lunar mission in real time. One of the features of the imaging system on the basis of a helmet - full overlap of the field of view of the operator and the substitution of the real environment by the virtual counterpart, which involves the use of tracking movements of an operator. 


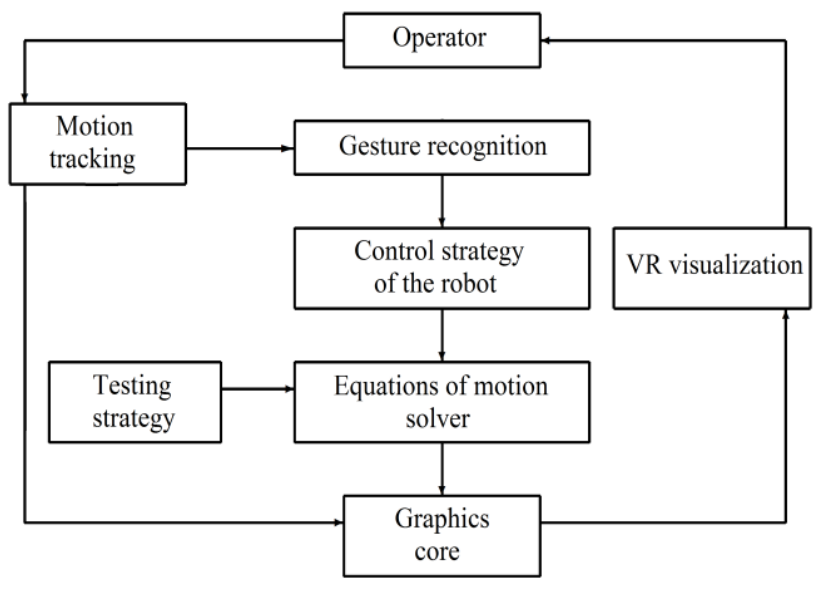

Figure 1. VR-stand structure

\section{TESTING METHODOLOGY THE PERFORMANCE OF THE CONTROL ALGORITHMS OF THE ROBOT IN EXTREME CONDITIONS}

Virtual reality stand, unlike full-scale simulation, involves in a laboratory experiment the creation of conditions contrast to the full-scale simulation involves the establishment the conditions, in which a cosmonaut has difficulty in dynamic adaptation to extreme conditions, and the study of collisions, which are caused by the actions of the cosmonaut. For this was developed by a specialized method Maximin (max-min) testing, in order to form the worst extreme factors in a simulated mission, as well as to compare the algorithm of automatic control of the robot with the optimal control on a number of criteria (Lemak, 2015). The technique is a Maximin test is designed as a tool intended to help instructors to composition, and allows to considerably varying the conditions of the mission in virtual reality.

\section{CONCLUSIONS}

In this article, we presented the analysis of methods of interaction in the team of cosmonauts and mobile robots in one of the possible modes of the EVA, when the operator on the lunar surface moves accompanied by a robot. This regime is useful not only in situations when the operations of exploration of the moon territory are carried out, but also in the cases of movement of cargo, installation of equipment and devices away from a lunar base, as well as during installation of navigational radio beacons.

The "Follow Me" mode is proposed as one of the preferred options for remote control of the robot in EVA. In laboratory studies, it is planned to verify the performance of proposed solutions with use of a simulation bench, which is equipped with a Toolkit of simulation of EVA conditions EVA and informational support of the interaction between its members (Kryuchkov et al., 2014; Burridge and Graham, 2001; Damian et al., 2013). A significant place in the planned research will be devoted to instrumental systems of motion capture (Stanton, Bogdanovych, Ratanasena, 2012) for studying human capabilities while performing gestural control in a simulated virtual environment (Karpov et al., 2016; Burridge and Graham, 2001; Damian et al., 2013).

Among the main ways to improve the human-robot interaction during a laboratory experiment on the simulation computer stand we study:
1) The use of mono and stereo cameras to detect the environment, the cosmonaut and his gestures. In particular, the space suit can have markings according to the principles of constructing a skeleton model.

2) The use inside the suit of "flexible" exoskeleton with embedded sensors of angular velocity. This will determine the relative positioning of the moving parts of the human shoulder girdle, which is necessary to identify the position of hands if optical sensors of the robot are covered.

3) The ability to assess cosmonaut-operator's movements by receiving and processing cosmonaut's shoulder girdle myogram in real-time.

4) Duplication of commands of the cosmonaut, performing EVA, by the dispatcher that monitors EVA remotely from the lunar lander; in the case of "master-slave" communication failure or incorrect execution of commands by the robot, the cosmonaut makes decisions on compliance with EVA safety requirements and the risk of collision.

5) Construction of a virtual environment, simulating EVA on the lunar surface, which includes 3D-models of a cosmonautoperator and a robot-assistant, for optimizing the cosmonautdispatcher's performance.

6) Evaluation of the results and safety of human-machine interaction in standard and emergency situations, simulated in the virtual environment, is done using mathematical methods.

Further laboratory testing is planned to organize a network of remote interactions in a virtual environment, including testing the lunar mission an international crew without the need of personal presence in the same room. Another promising option is the use of remote high-performance computing complex for the testing of the mathematical model of the entire lunar missions and calculate the testing strategies, while on the local virtual reality system will be modeled only the area within easy reach of the operator. This will significantly optimize the resources of the virtual reality booth, designed for high-quality visualization.

\section{ACKNOWLEDGEMENTS}

This work was partially supported by a grant from the RSF 1450-00029 in the development of the virtual reality stand.

\section{REFERENCES}

Vorona, A., Syrkin, L., Kryuchkov, B., Usov, V., 2016. Visual Displaying a Group of Autonomous Mobile Robots Moving on the Moon's Surface in Order That a Cosmonaut Could Prevent Their Collisions. Manned Spaceflight, 20(3). pp. 41-57. (In Russian)

Karpov, A., Kryuchkov, B., Ronzhin, A., Usov, V., 2016. Designing human-robot interaction in a united team of cosmonauts and autonomous mobile robots on the lunar surface. In Proc. 26th International Conference «Extreme Robotics (ER2016)», St. Petersburg, Russia, pp.71-75.

Kryuchkov, B., Karpov, A., Usov, V., 2014. Promising Approaches for the Use of Service Robots in the Domain of Manned Space Exploration. SPIIRAS Proceedings, 32(1), pp. 125-151, doi:10.15622/sp.32.9 (In Russian)

Kryuchkov, B., Mikhailyuk, M., Usov, V., 2015. Information support of a cosmonaut during visual inspection of the 
movements of mobile anthropomorphic robot in zero gravity. In Proc. 8th All-Russian Multi-conference on Management Problems (MCPU-2015). The conference "Robotics and Mechatronics" (RoMe-2015), vol. 2. pp. 248-250. (In Russian)

Burridge, R. and Graham, J., 2001. Providing robotic assistant during extra-vehicular activity. Intelligent Systems and Advanced Manufacturing / Mobile Robots XVI.

Damian, I., Obaid, M., Kistler, F., André, E., 2013. Augmented reality using a 3D motion capturing suit. In Proc. 4th Augmented Human International Conference AH-2013, pp.233234. doi:10.1145/2459236.2459277

Fong, T., Nourbakhsh, I., Dautenhahn, K., 2016. A survey of socially interactive robots. Robotics and Autonomous Systems, 42(3-4), pp. 143-166.

Liu, J., Luo, Y., Ju, Z., 2016. An Interactive Astronaut-Robot System with Gesture Control. Computational Intelligence and Neuroscience, article ID 7845102, 11 pages. doi:10.1155/2016/7845102

Stanton, C., Bogdanovych, A., Ratanasena, E., 2012. Teleoperation of a humanoid robot using full-body motion capture, example movements, and machine learning. In Proc. Australasian Conference on Robotics and Automation, Victoria University of Wellington, New Zealand.

Lemak, S., 2015. Formation of positional strategies for a differential game in Krasovskii's method of extremal aiming. Moscow University Mechanics Bulletin, 70(6), pp.157-160, doi:10.3103/S0027133015060059 